\title{
Optimization of a Jet Engine Compressor Disc with Application of Artificial Neural Networks for Calculations Related to Time and Mass Criteria
}

\author{
Adam Kozakiewicz' ${ }^{1}$ Rafał Kieszek ${ }^{1 *}$, Robert Rogólski ${ }^{1}$ \\ 1 Military University of Technology, Faculty of the Mechatronics, Armament and Aerospace, Institute of Aviation \\ Technology, ul. S. Kaliskiego 2, 00-908 Warszawa, Poland \\ *Corresponding author's email: rafal.kieszek@wat.edu.pl
}

\begin{abstract}
The paper presents the results of a series of numerical research on the possibility of applying Artificial Neural Networks (ANNs) for the ultimate strength calculations of selected parts of rotating machines. The layout and the principle of the algorithm operation were described, beginning from the general assumptions and then moving on to the detailed description of the subsequent modules. The effects of applying the algorithm were presented on the example of the compressor disc analysis. The significant benefits of its application were the reduction of optimization time by about $40 \%$ and the disc weight reduction by $0.5 \mathrm{~kg}$. The accuracy of ANNs was different in each iteration of the presented algorithm. Finally, high accuracy of neural networks was achieved with the following mean values of relevant indices reached in the last iteration: $R M S E=0.5983, M A P A=0.0733$ and $R^{2}=0.99895$. The further perspectives of the undertaken research were defined at the end.
\end{abstract}

Keywords: aerospace engineering, artificial neural networks (ANNs), optimization, turbine jet engines, axial compressors.

\section{INTRODUCTION}

Reasonable and efficient implementation of Artificial Neural Networks to tasks of mechanical engineering, in particular aerospace engineering, is a significant future challenge for science. For example in papers $[1 \div 5]$, authors used ANNs to predict the material strengths and the methodology dedicated to that problem was described in details. In the works from the last few years, there are indeed interesting solutions for mechanical problems but there are no implementations of ANNs in aerospace engineering, especially in design and optimisation components of jet engines.

In the paper [1] hybrid ANN and bee colony algorithm were applied to predict the FRP-concrete bond strength evaluation. The database contains 656 cases collected from literature where it were experimentally determined. Similarly, in the paper [2] Feed Forward Back Propagation Neural Network was used for the prediction procedure to evaluate the compressive strength of concrete elements. The training database was also taken from the literature.

Abdulla [3] conducted the analysis of Concrete-Filled Plastic Tube by ANNs formulating an empirical expression for predicting the axial compression capacity and axial strain. In the same paper [3], the compressive strength of FRP-confined circular reinforced concrete columns was predicted with the use of algorithm based on ANN.

Prediction of the compressive strength of FRPconfined circular reinforced concrete columns has been performed in the paper [4]. Artificial Neural Networks, Group method of data handling and Gene expression programming were comprised. The highest accuracy was achieved by ANNs.

In the paper [5], the compressive strength of IBP-Based Geopolymer Stabilized Clayey Soils 
was determined by two soft computing algorithms: the neural network-based group method of data handling (GMDH-NN) and artificial neural network (ANN). High accuracy of both methods was achieved. The authors stipulate that the equations of GMDH-NN are not so complicated as ANN, but the accuracy of ANNs is higher. The $R^{2}$ values are 0.98 and 0.99 for training and 0.96 and 0.97 for testing of GMD'H-NN and ANN models.

The authors analysed the papers in which algorithms based on ANNs were mostly applied to predict strength in models where: experiments are expensive, time consuming or complicated to calculate by computer simulation. The analysed literature shows that ANNs are especially useful and efficient when are applied in combination with other software algorithms like bee colony [1] or the gene expression algorithm [3].

In the area of aircraft propulsion design, the problem to optimize the construction of a compressor unit is one of the most important engineering problems. This fact can be proven by the changes in the design of engine compressors according to data of one of the engine manufacturers over a period of 15 years, as shown in Fig. 1. The complex process of optimizing the construction of compressors and fans of turbine engines should include the following aspects:

- reduction of the number of stages and reduction of part weights with the preserved parameters of the sub-assembly;

- improving the stability of the compressor operation;

- increasing the efficiency of bladed-row palisades by implementing the radial curvature in blade-contour profiles;

- increasing in the heat durability of structural materials;

- more widespread use of materials with monocrystalline structure and the BLISK technology;

- reducing the need for relative air intake from the compressor, which is necessary to cool other engine components;

- increasing the bypass ratio for inside channel flow;

- extending the life cycle of rotor components.

In the above-mentioned engineering problems, the increasing calculation time is one of the most crucial problems faced by working teams of engineers. the weight optimization of the compressor disc taking into account its ultimate strength can be mentioned as an example of a time-consuming engineering problem.

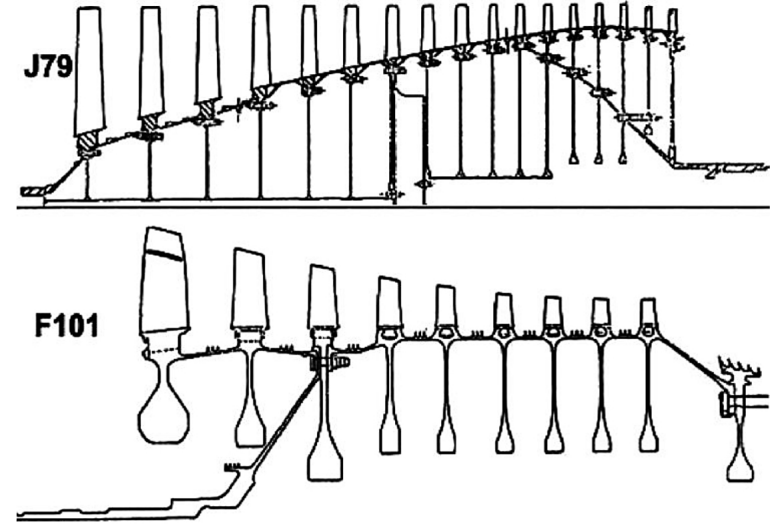

Fig. 1. Schematic diagrams of compressors of the GE J79 (first run in 1955) and GE

F101 (first run in 1970s) jet engines [12]

In order to reduce the calculation time, the application of Artificial Neural Networks (ANNs) was proposed. In aviation, neural networks are primarily used for broadly defined identification, simulation and optimization [6]. They are also increasingly used in the design process [7], and in recent years ANNs are being combined with hard computing techniques (such as FEM - Finite Element Method or FVM - Finite Volume Method) for standard numerical calculations $[8 \div 11]$.

Scientific publications in the field of aircraft engine optimization usually deal with the problems of optimization of the blade shape [13, 14], where usually the objective function is the efficiency, or shortening the spiral design, like in [15], where the compressor is only one of the subassemblies of the designed engine. An objective function in the paper [13] was defined according to the expression below:

$$
\begin{aligned}
f(X) & =50 \cdot \eta_{1}+100 \cdot \eta_{2}+50 \cdot \eta_{3}+ \\
& +10^{4} \cdot \max \left(1.45-\pi_{1}, 0\right)+ \\
& +10^{4} \cdot \max \left(1.65-\pi_{2}, 0\right)+ \\
& +10^{4} \cdot \max \left(1.73-\pi_{3}, 0\right)+ \\
& +100 \cdot \max (\operatorname{mf} 1-34.25,0)+ \\
& +100 \cdot \max \left(34.15-\mathrm{mf}_{1}, 0\right)
\end{aligned}
$$

where: $\eta_{1}, \eta_{2}, \eta_{3}$-efficiencies at high, intermediate, and low mass flow (partial objective functions), $\pi_{1}, \pi_{2}, \pi_{3}$ - total pressure ratio at high, intermediate, and low mass flow (constraints), $m f_{1}$ - mass flow at the high-mass flow operating point (another constraint).

In other way, Lee and Kim [14] define efficiency according to the following formula:

$$
f=1-\eta=1-\frac{\left(\frac{P_{\text {oexit }}}{P_{\text {oinlet }}}\right)^{\frac{\gamma-1}{\gamma}}-1}{\frac{T_{\text {oexit }}}{T_{\text {oinlet }}}-1}
$$




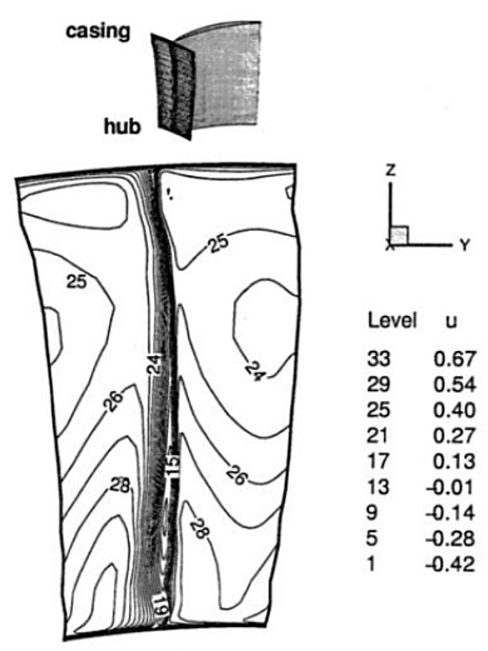

(a) Initial

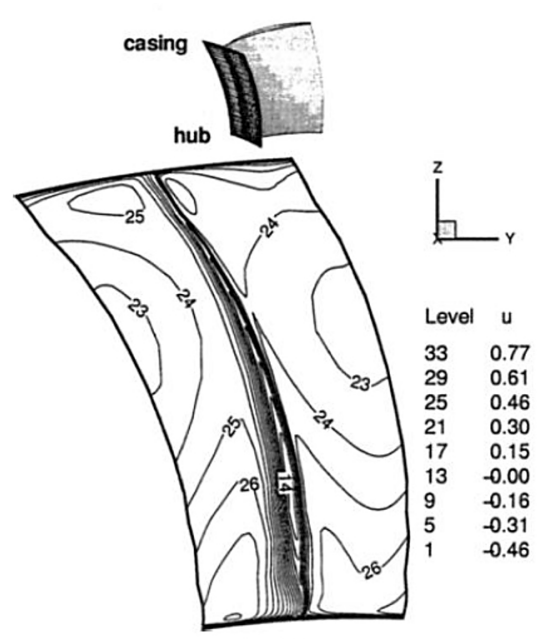

(b) Final

Fig. 2. Distribution of the absolute axial component of the Mach number (denoted with $u$ ) at the output from the rim of the guide ducts [14]

where: $p_{\text {oinlet, }} p_{\text {oexit }}-$ pressures at inlet or outlet of a single flow section, $T_{\text {oinlet }}, T_{\text {oexit }}$ - temperature at inlet or outlet, $\gamma$ - heat capacity ratio.

Apart of the one mentioned above, some other type of engineering problems can also be formulated. For example, in the research paper [14] one finds the algorithm for comparative numerical analysis of the optimized vanes of compressor cascade. The iterative algorithm was used to solve the Navier-Stokes equation system, bringing the output closer to the minimum of the objective function, which the efficiency was assumed to be. As a result of the optimization, the vanes with a significant curvature of the blade tip and a more favourable distribution of Mach number were obtained (Fig. 2).

A similar optimization process was also described in details in the research papers [16] and [17]. The authors of the first research paper present the process of designing and optimizing an axial compressor of a small, transonic jet engine as an implementation ANNs to maximize thrust and minimize unit fuel consumption. For that purpose, the problem of replacing the axial compressor with a radial one was considered. Radial compressors are mainly used in the engines with a low mass flow ratio. In both cases, the design and optimization process itself of axial and radial compressors are similar, apart from the design differences. The combination of numerical and experimental research has shown that for small engines it is more advantageous to use a diagonal compressors than purely radial ones. The paper [17] contains an algorithm to optimize a multi-stage axial compressor, where Artificial Neural Networks were used to estimate losses and deviations in the process of designing blade airfoils, which allowed designing a new family of blades with optimized section shapes (Fig. 3). A similar problem of turbine optimization is presented in the paper [15].

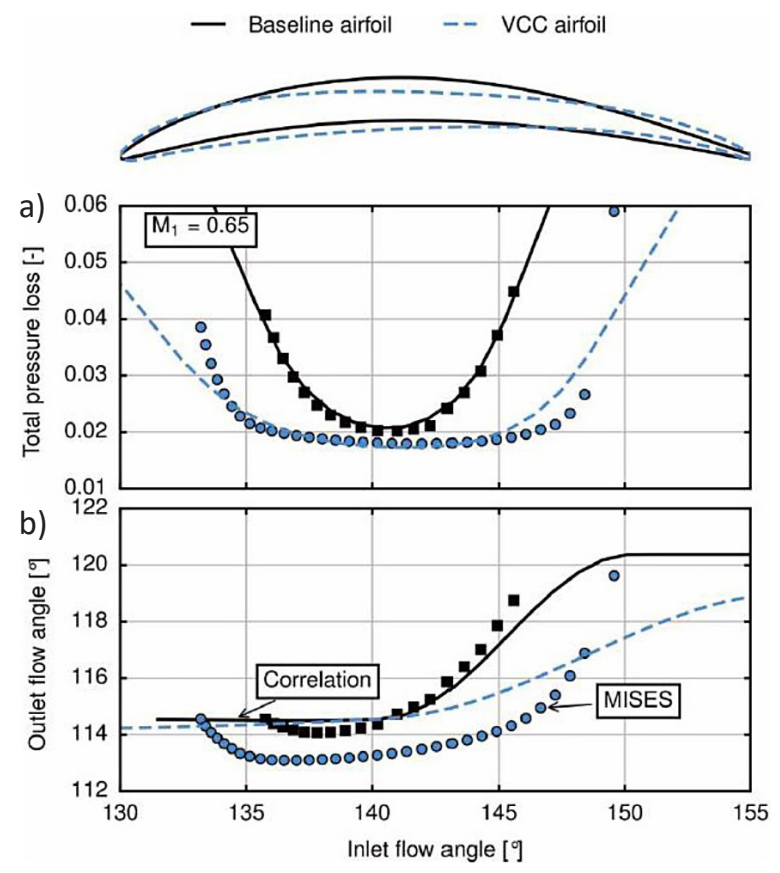

Fig. 3. Results of compressor blade geometry optimization, where: a) pressure loss factor, b) deflation angle, depending on the attack angle for the output profile (baseline airfoil) and after optimization (VCC airfoil) [17] 


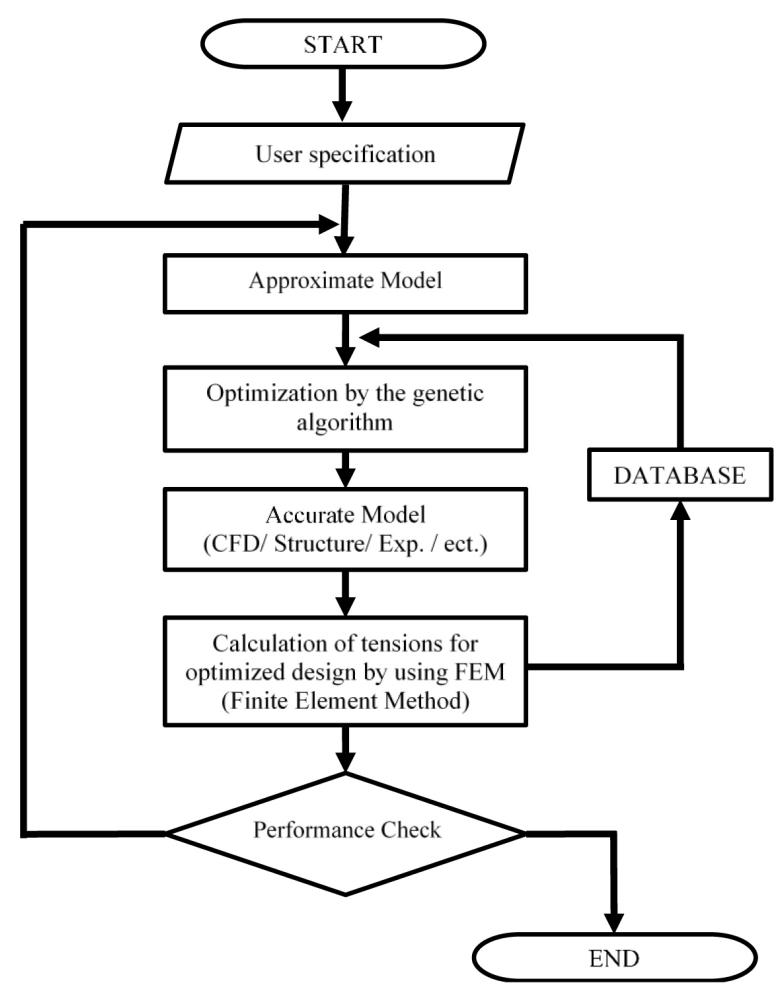

Fig. 4. Flowchart of optimization strategy [13]

The algorithm to optimize the operation point and shape of the blades of the compressor cascades, with an algorithm including both a hard (FVM) method and a soft (genetic) technique, were described in the study [13]. The flowchart of the proposed algorithm is shown in Figure 4.

The first part of the algorithm includes data preparation and in the analysed case, the properties of NASA 67 rotor were used. Then, an approximate model was created in order to build an analytical relationship between the design variables and the simulation results. In the next step, an approximate optimization with a genetic algorithm was performed on the basis of a previously developed analytical model. The objective function is a polynomial containing a compression factor and a stage efficiency index depending on the acceptable flow rate values. The next step was to validate the results with a thorough simulation, which complements the database of the input data. The computer program repeats the above-mentioned steps from creating an approximate model to reaching the stop condition. The numerical process is terminated by achieving the assumed accuracy or after carrying out an assumed number of iterations.

As result of 120 iterations, the optimization algorithm increased the efficiency of the compressor cascade by $0.5 \%$, due to the optimized blade geometry (Figure 4). The conclusions presented by the authors concerned the algorithmic operation time, which can be reduced by the approximation of an applied model including its update every few time steps. To some extent, it is possible to automate optimization by imitating the designer's intelligent behaviour. The algorithm operation time was about a week. In comparison, the same problem can be solved by an experienced team of people within two to three weeks.

Taking into account the referenced papers, it could be noticed that the process of optimization of the axial compressor disc using ANNs in order to calculate the stress distribution for the time and mass criteria was not encountered so far. Therefore, the method and results presented in this paper could be treated as a quite new and original proposition. The algorithm presented here enables an optimization of structural components with the example of mass optimization of the axial compressor disc by using ANNs in shorter time, comparing to the classical approach. This research work is the extension of previous research works, where the authors use ANNs to calculate the stresses distribution in the compressor disc [18].

\section{STRUCTURE OF THE ALGORITHM}

The developed algorithm is supposed to optimize the shape of the disc of the jet engine axial compressor, taking into account the time criterion. The objective function of the algorithm is to minimize the mass of the disc taking into account the ultimate strength. One of the important problems in solving this type of tasks is the time consumption of a numerical process.

By using the finite element method (FEM) techniques available in commercial software (e.g. ANSYS, Nastran), it is possible to prepare a preoptimized disc model and then analyse it to calculate the distribution of stresses. The computer program either automatically removes the redundant elements or, less often, the operator does it. Then, the surface of the optimized object is smoothed and the process of numerical calculations and removing unnecessary elements is repeated until a satisfactory result is achieved. It should be noted that in this case, the algorithm cannot add material to reduce the load on other parts of the structure element. Both processes of stress calculations 
and FE model reduction are relatively long and increase along with the number of iterations and accuracy. The presented solution is a kind of optimization with the use of Monte Carlo method. Figure 5 presents the general structure of the algorithm, where a significant part of calculations in traditional approach performed by FEM is replaced by the ANN-based algorithm. A similar algorithm was used in the paper [13], with a different approximation model and genetic algorithm. The approximation model used in that paper [13] is based on the radial neural networks, whereas in this research, the Feed Forward-type Artificial Neural Networks were used. Additionally, in the study [13], the size of database increases with each iteration unlimitedly, while in the algorithm proposed by the authors, the size of the database is limited to a maximum of 50 learning cases. When the algorithm generates 10 more training cases in the loop, the oldest 10 are deleted. Limited size of the learning database prevents from increasing the training time, while accuracy increases in every loop of algorithm.

After entering the input data into the computer program, the stresses distribution necessary for ANNs to learn is calculated. Then, the computer sub-program, using a genetic algorithm, optimizes the mass of the disc, taking into

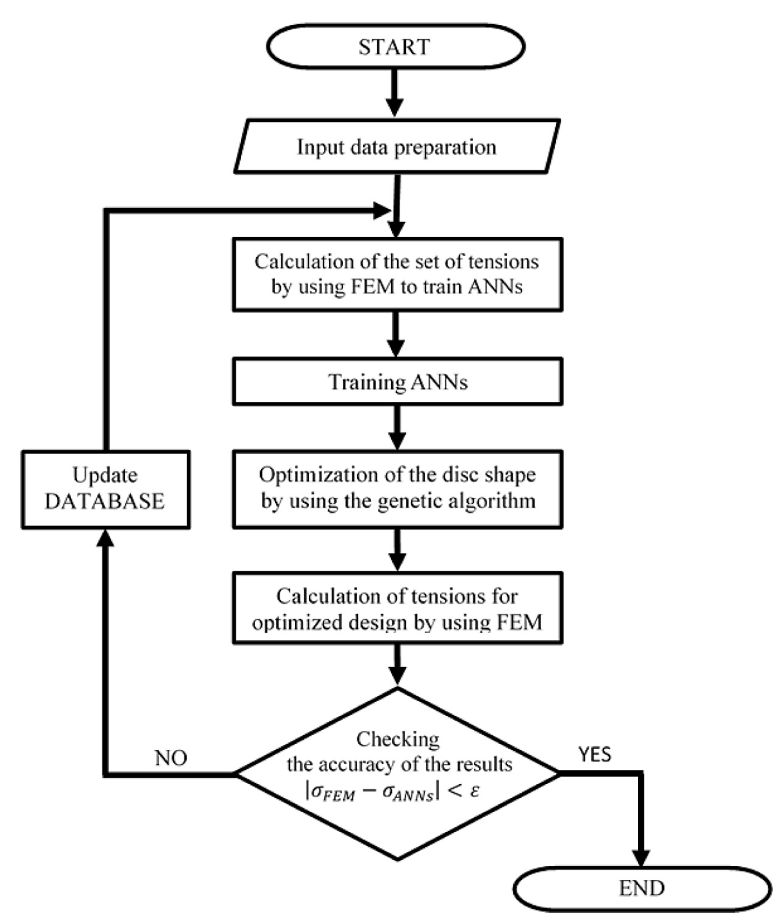

Fig. 5. Flowchart of the algorithm for optimization of compressor disc account the ultimate stress condition. In the next phase, FEM calculations are performed, and then some control calculations are carried out to verify the accuracy of the ultimate strength condition against ANNs. If this accuracy is unsatisfactory or if the results differ from the optimal results from the previous iteration, the learning of ANNs is resumed and ANNs are optimized again. In order to validate the time criterion, the time to generate data necessary to train ANNs and the time of operations by the genetic algorithm are subjected to measurement.

\section{MAIN PROCEDURES OF THE ALGORITHM}

The general structure of the algorithm was presented in the previous section. This part of the paper will discuss in detail the main procedures of the developed algorithm such as: the type of input data, the applied FEM algorithm, the structure of the ANN, the objective function and the penalty function, and the limitations of the genetic algorithm.

The computer program input data include the following parameters:

- material properties (Young's module, Poisson's number, disc material density),

- outer radius of the disc and the radius of the hole inside the disc,

- angular velocity of the disc,

- mass of the blade with its dovetail and with the disc fragment necessary to fulfil the SaintVenant principle,

- total number of blades,

- radius of position of the weight centre of a single blade with its dovetail part,

- maximum and minimum acceptable disc thickness.

Additionally, 18 disc thickness values on standardized radius positions (according to the equation 3) were introduced. The thickness at the base of the disc has been imposed as the maximum acceptable value. Initially, the disc was divided into 10 equal finite elements. Then, the first two elements were divided into four elements, and the last two into two, which resulted in a disc divided into 18 finite elements with refinement mesh around the edges, again according to equation 3. In the case of a disc of any size, the position of successive nodes always corresponds to the same relative radius, which enables the parameterization of various discs in order to train the artificial neural networks. 


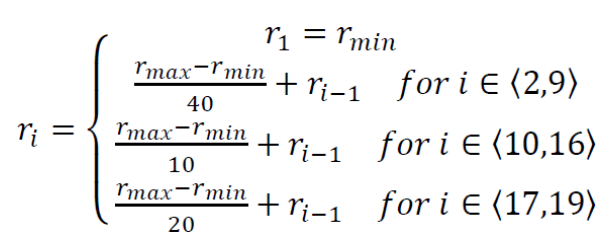

where: $r_{\min }$ - radius of the disc hole, $r_{\max }$ - outer radius of the part of the optimized disc

All data entered into the algorithm (including the material data) were declared in matrix form, which enables to take into account the change of material properties, e.g. in case these properties are dependent on temperature. In figure 6 , input and output parameters necessary to form the ANN process were shown.

The results obtained from the FEM-based algorithm were validated by comparing them with the analytical calculations for the straight disc and with the results of the commercial computer program (ANSYS) for the profiled disc. The difference between the stresses calculated from of the FEM algorithm (simplified by omitting the matrix of the additional stiffness) and the stresses obtained for an optimized disc does not exceed $0.5 \%$.

In order to prepare the data for the ANN learning process, the set of discs was drawn from the range $\pm 5 \%$ of the disc thickness relative to the last optimal solution. If the disc thickness was higher on the next radius (counting from the axis of rotation) than on the previous radius, it was replaced by the value from the previous radius. This enabled to obtain a thickness distribution consistent with the real objects. The maximum and minimum acceptable disc thickness values were also checked.

In the next phase, the matrix of the learning cases was calculated. It was used to generate the proper input and output data. The algorithm for 10 cases from a previously prepared matrix calculates the stresses distribution using the finite element method, which approximates the disc with conical shell elements having linearly variable thickness. The case study with similar elements was described in more detailed way in the paper [19]. In case of numerical problems related to disc with extremally high thickness gradient, the case is overwritten by another one. In each loop of the algorithm, the new 10 cases are added to those ones already prepared in previous iterations and the oldest 10 are deleted. The computer program has been configured so that the database trains only 50 learning cases at one time. This allows training ANN in a fast and sufficiently accurate way and to generate completed data for the necessary database. Finally, the computer program sends data to the next computer sub-program.

In order to determine the structure of the Artificial Neural Network and the selection of the learning algorithm, numerical research was conducted, which resulted in selecting the Feedforward-type network. The NARX network (nonlinear autoregressive neural network with exogenous inputs) was also considered, but its learning time was longer with lesser accuracy. Then, a comparative analysis of 9 learning algorithms was performed, including:

- Scaled conjugate gradient backpropagation (SCG),

- Levenberg-Marquardt backpropagation (L-M),

- Gradient descent with momentum and adaptive learning rate backpropagation (GDA),

- BFGS quasi-Newton backpropagation (BFGS),

- Gradient descent with momentum backpropagation (GDM),

- One-step secant backpropagation (OSS),

- Conjugate gradient backpropagation with Powell-Beale restarts (CGB),

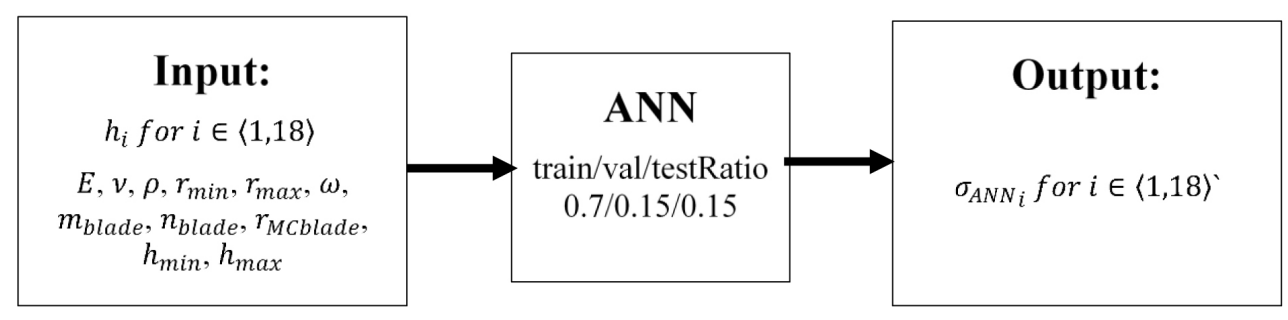

Fig. 6. ANN input-output parameters with training, testing and validation ratio of training, where: $h_{i}$ - thickness of disc for every normalized radius, $E$ - Young's modulus, $v$ - Poisson's ratio, $\rho-$ disc material density, $r_{\min }$ - radius of the hole inside the disc, $r_{\max }-$ outer radius of the disc, $\omega-$ angular velocity, $m_{\text {blade }}$ - mass of the blade, $n_{\text {blade }}$ - number of blades, $r_{\text {MCblade }}$ - radius of position of the centre of the blade mass, $h_{\min }$ - minimum acceptable disc thickness, $h_{\max }$ - maximum acceptable disc thickness and $\sigma_{A N N_{i}}$ - reduced stress value calculated by ANN for every normalized radius 
Table 1. Network efficiency depending on the number of hidden layers

\begin{tabular}{|c|c|c|c|c|c|}
\hline Hidden layer & $\delta[\%]$ & $\mathbf{t}[\mathbf{s}]$ & $\delta[-]$ & $\mathbf{t}[-]$ & $\mathbf{n}[-]$ \\
\hline 2 & 10.37 & 0.84 & 1 & 1 & 1 \\
\hline 3 & 13.23 & 1.18 & 0.783 & 0.7119 & 0.558 \\
\hline 4 & 7.24 & 0.97 & 1.432 & 0.8660 & 1.240 \\
\hline 5 & 6.01 & 1.22 & 1.726 & 0.6885 & 1.188 \\
\hline
\end{tabular}

- Conjugate gradient backpropagation with Fletcher-Reeves updates (CGF),

- Conjugate gradient backpropagation with Polak-Ribiére updates (CGP).

The ANN learning method was tested in two ways based on acceptable disc thickness values. The first way was based on $(100 \pm 2) \%$ of a disc thickness, the second way was based on $(100 \pm 5) \%$. The accuracy of the model was measured by percent error of von Mises stresses calculated for ANNs and FEM stress numerical values. In the first case $(100 \pm 2) \%$, the SCG, L-M, BFGS algorithms learned much longer $(24,79,126$ seconds on average), the others under $1 \mathrm{~s}$, and all with comparable accuracy between the algorithm for results of both analysis training and testing database for the compared algorithm. However, in the case of $\pm 5 \%$ data, only the SCG algorithm was able to train the network with the selected architecture. It was also verified that training the network twice with the chosen method significantly reduces the probability of occurrence of serious training errors, which, in the case of a single training, occurred in about 1 out of 5 cases, while in case of a double training, serious errors were not found. The accuracy was calculated as mean error of stresses computed by ANN and FEM algorithm.

All calculations were performed on a $\mathrm{PC}$ equipped with Intel CORE i5-9300H $2.40 \mathrm{GHz}$, GeForce GTX 1650, 8 GB RAM. The applied software was MATLAB R2018a with the Neural Network Toolbox 11.1. The number of layers in the network was selected on the base of the learning efficiency of the network $\eta$ which was the product of reduced relative error and reduced time parameters (eq. 4).

$$
\eta=\frac{\delta_{1}}{\delta_{i}} \frac{t_{1}}{t_{i}}
$$

where: $\eta$ - learning efficiency of the network, $\delta_{1}$ - relative error of the first network, $\delta_{i}-$ relative error of the i-th network, $t_{1}$ - training time of the first network, $t_{i}-$ training time of the i-th network
Networks of two to five hidden layers, 50 neurons in each layer, were studied. The results of the analysis are presented in the table (the best result marked with bold).

A neural network with four hidden layers was chosen because it had the highest efficiency. The initial training error was large, but it was reduced to about $1.5 \%$ by cyclic operations of the whole algorithm, in which the network is still being trained several more times while modifying the training case database. The ANN with a single hidden layer had too many errors of matching; therefore, it has been skipped in Table 1 .

The number of neurons was taken by checking the values every 20 neurons per layer (starting from 10). For values of 30 neurons and less, the network had a tendency to learn by heart, especially in the case $\pm 2 \%$ of the disc thickness. For networks with more neurons, the training time increased significantly with a small increase in accuracy. For 70 neurons, errors and time of calculation were soaring; therefore, the analysis with inclusion more number of neurons in layer has been abandoned.

The next computer sub-program is used only to train ANNs (Fig. 7). The algorithm, after previous numerical analysis, uses Feedforward-type Artificial Neural Networks implemented in MATLAB computer program using Neural Network Toolbox. The network was composed of four hidden layers, 50 neurons in each layer, and one output layer. Each single neuron has the biases and the sigmoid activation function.

The input data for the network are the 24 parameters mentioned above. The only output parameters are the stress values. In total, 18 Artificial Neural Networks (Feedforward-type) were used, each of which calculated stresses in one of the previously dimensioned elements. The network was trained by using the SCG (Scaled Conjugate Gradient method) described in detail in the paper [20], which, similarly to the Coupled Gradient method, is derived from developing the objective function into Taylor's square equations and omitting small ones of the higher order. The difference 


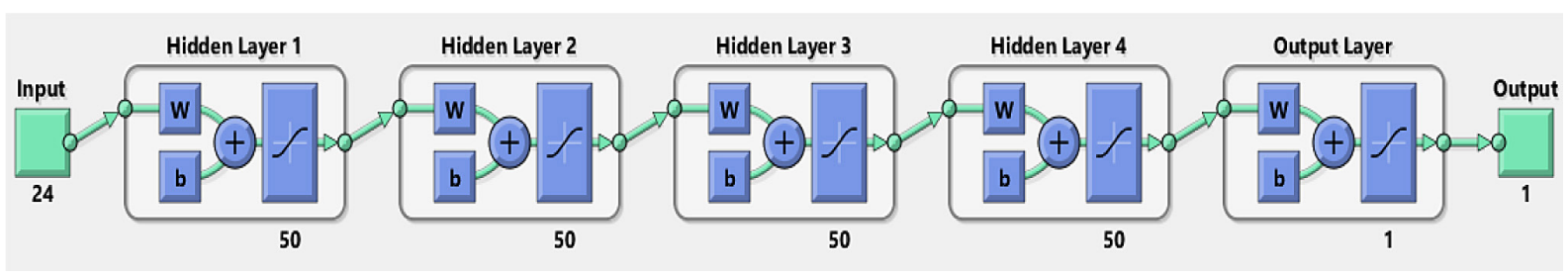

Fig. 7. The ANN scheme for disc stress calculations, where: Input 24 - input data; Hidden Layer 1, 2, 3, 4 - hidden layers, 50 neurons in each layer; Output Layer - a single neuron of the output layer from the network; Output 1 - parameter to output from network

lies in the combination of the simultaneous calculation of the conjugated search direction and the optimal step in this direction. The training process continued until no improvement was achieved in 50 consecutive iterations and was repeated twice for each network. The learning time for all ANNs within one iteration of the whole proposed algorithm operations is about 40 seconds.

The disc shape optimization computer subprogram was used to optimize the thickness in 18 nodes (external nodes), to which the genetic algorithm was applied. The thickness of the disc, for the node corresponding to the inner edge of the disc has been set. The upper and lower limits were adopted as for the thickness of the discs prepared for learning and they represented $\pm 5 \%$ of the thickness of the optimal disc from the previous iteration. Another limitation was the thickness of the disc. The thickness of the next radius cannot be thicker than that of the previous one, counting from the symmetry axis of the disc solid.

Genetic algorithm used stochastic uniform selection. Variable space was limited similarly as size of database. Maximum time was limited to 2100 seconds and maximum number of generations was limited to one, due to generate new training database in order to increase the accuracy of the algorithm. The objective function in the algorithm was to minimize the mass with regard to the ultimate strength. The ultimate strength condition was taken into account in the form of the penalty function (eq. 5).

During the operations, the genetic algorithm generates new cases of discs and calculates their masses, checking the ultimate strength for the whole disc (using the already learned ANNs). In case of exceeding the ultimate stress value, the penalty calculated as a square of one thousand times the difference between the stresses calculated in each element and the maximum acceptable (ultimate) stresses, was added to the mass resulting from the geometry. This enabled to switch from the objective function with restrictions to the objective function with no restrictions but with the penalty. The value of the penalty was chosen experimentally so that a sharp increase in the value of the function beyond the acceptable limits of the area is ensured with the condition fulfilled that the derivative of the objective function is continuous.

$m=\left\{\begin{array}{cc}V \rho & \text { if no penalty } \\ V \rho+\left|\sigma_{A N N}-\sigma_{\text {allow }}\right| \cdot 10^{6} & \text { if penalty }\end{array}\right.$

where: $V$-volume of the disc, $\rho$-material density, $\sigma_{A N N^{-}}-$stress calculated by ANN, $\sigma_{\text {allow }}{ }^{-}$ maximum allowable (ultimate) stress.

Each generation was followed by the validation of the accuracy of the algorithm using FEM. If the ANN algorithm operation was accurate and the optimal solution did not change significantly from the last one, the algorithm was stopped. Otherwise, there was a return to generating the data necessary to train ANNs in the newly limited area of acceptable solutions.

The operation time of the genetic algorithm has been limited to one generation during the operation of the optimization computer sub-program, although longer calculation time may increase the accuracy of the solution by bringing it closer to the global minimum. This limitation was applied because the penalty is calculated on the basis of the ANN algorithm, which has finite accuracy, the smaller accuracy the closer to the boundary of the solution space (which the ANN has learned earlier) the calculated case is located. Interrupting the searching for the minimum and the complementary training of ANNs for the new cases from the area of the last optimal solution significantly reduces the time and number of iterations of the genetic algorithm operations necessary to achieve the minimum with the required accuracy.

The optimization algorithm based on the ultimate strength criterion is checked with taking into account the FEM results. If the stress condition is not fulfilled, the loop is repeated. 
a)

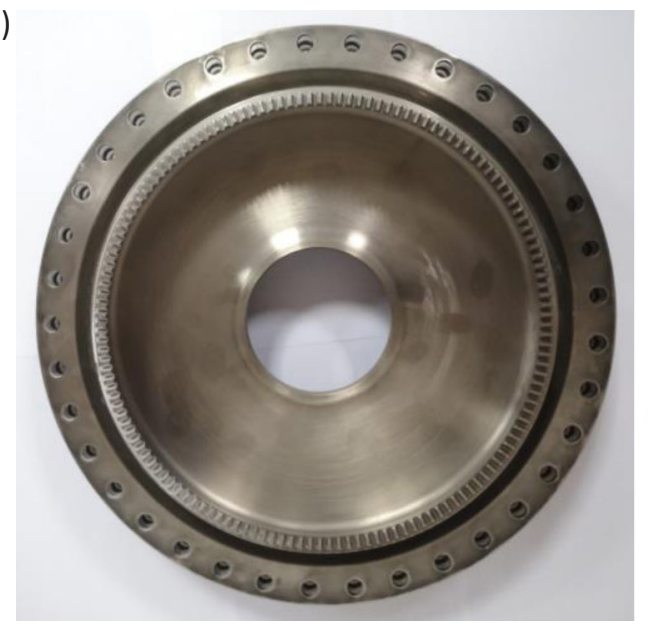

b)

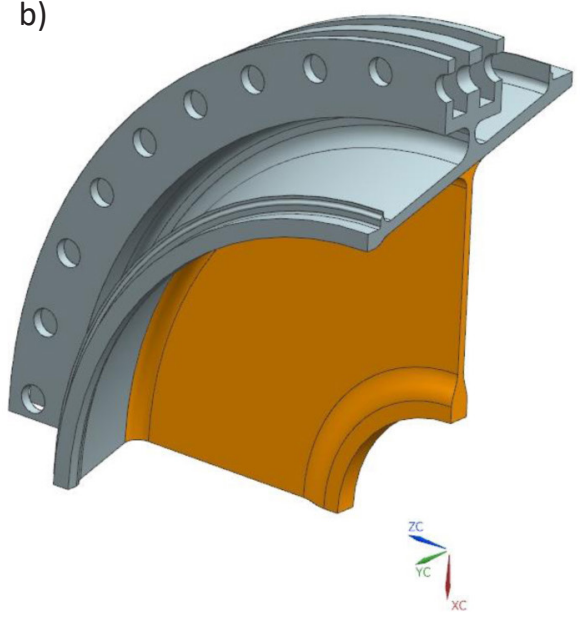

Fig. 8. The actual object (a) and the reference geometric model of the disc with marked optimized area (b)

\section{COMPRESSOR DISC OPTIMIZATION}

The optimization process was carried out for the inner part of the disc marked in orange in Figure 8, using the algorithm presented in the previous section. Optimization of this structural system has also enabled to refine the algorithm.

Five iterations were performed during the optimization process. The accuracy of ANNs output specific parameters, obtained after last iteration of genetic algorithm was presented in Table 2 .

Mean value of accuracy of all networks reaches the following values: $R M S E=0.5983, M A P A=$ 0.0733 and $R^{2}=0.99895$. High accuracy of neural networks has been achieved.

One iteration lasted about 98 seconds. The algorithm was preparing the input data for about $40 \mathrm{~s}$ and the rest of time has been consumed on the learning and training Artificial Neural Networks. The results of the process of optimization of shape and the stresses distribution are shown in Figure 8.

The stresses calculated for the FE model $\sigma_{F E M}$ shown in Figure 9 were calculated in the last stage of the algorithm operation. The mass achieved of the optimized disc (Fig. 10) is about $7.6 \mathrm{~kg}$. As a result of the optimization process, the mass of the disc was successfully reduced by 0.5 $\mathrm{kg}$ comparing to the actual disc.

The optimization time was compared with an identical algorithm based solely on FEM, hereinafter referred to as the reference algorithm. In this algorithm there were no blocks related to the training of ANNs and related to generation of the training cases. In the objective function, the penalty was applied based on the stresses calculated from the FEM algorithm. The operation time of the whole reference algorithm is about $170 \mathrm{~s}$. The time to prepare the input data for the algorithm in this case is about $3 \mathrm{~s}$, and the remaining time is devoted to the operations by the genetic optimization algorithm. Figure 11 shows a comparison of the operation time of the proposed algorithm which uses ANNs and the FEM reference algorithm.

Table 2. RMSE, MAPE and R2 for ANNs

from the last iteration of the algorithm

\begin{tabular}{|c|c|c|c|}
\hline Number of ANN & RMSE & MAPE & $\mathbf{R}^{\mathbf{2}}$ \\
\hline 1 & 1.3041 & 0.1242 & 0.99915 \\
\hline 2 & 1.2487 & 0.1099 & 0.99890 \\
\hline 3 & 1.1778 & 0.1213 & 0.99904 \\
\hline 4 & 1.0996 & 0.1312 & 0.99924 \\
\hline 5 & 1.0478 & 0.1256 & 0.99916 \\
\hline 6 & 1.0340 & 0.1414 & 0.99910 \\
\hline 7 & 0.5993 & 0.0811 & 0.99914 \\
\hline 8 & 0.5274 & 0.0782 & 0.99923 \\
\hline 9 & 0.4842 & 0.0669 & 0.99915 \\
\hline 10 & 0.3630 & 0.0525 & 0.99908 \\
\hline 11 & 0.3010 & 0.0440 & 0.99897 \\
\hline 12 & 0.2593 & 0.0400 & 0.99890 \\
\hline 13 & 0.2439 & 0.0397 & 0.99876 \\
\hline 14 & 0.2446 & 0.0348 & 0.99850 \\
\hline 15 & 0.2109 & 0.0324 & 0.99881 \\
\hline 16 & 0.2169 & 0.0325 & 0.99859 \\
\hline 17 & 0.2030 & 0.0325 & 0.99869 \\
\hline 18 & 0.2046 & 0.0323 & 0.99870 \\
\hline Mean value & 0.5983 & 0.0733 & 0.99895 \\
\hline & & & \\
\hline
\end{tabular}




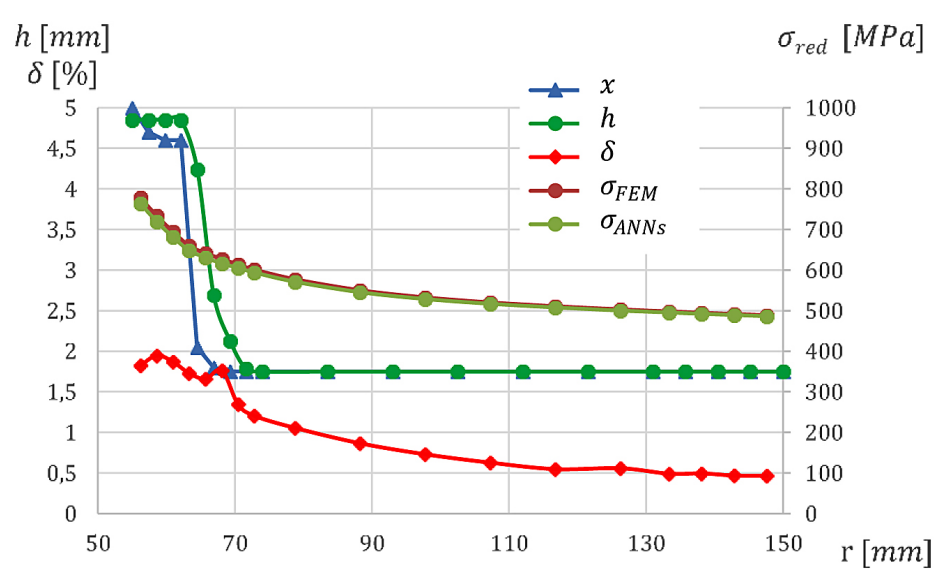

Fig. 9. The shape of the optimized disc and its corresponding distribution of reduced tensions, where $x$ - half the thickness of the optimized disc; $h$ - profile of the actual disc; $\delta$ - relative error of stresses; $\sigma_{F E M}$ - stresses calculated with FEM; $\sigma_{A N N s}-$ stresses calculated with ANNs; $r$ - radius of the disc

The proposed algorithm combining ANNs and the genetic algorithm operates relatively quickly. The algorithm operation time is about 7-8 minutes from the start of calculations. This time, in the case of the analysed problem, it included 5 iterations. The operation time of a single iteration of the whole reference algorithm is about $170 \mathrm{~s}$. The operation time of one iteration of the proposed algorithm based on ANNs is about $98 \mathrm{~s}$. The difference in time in the presented case is about $70 \mathrm{~s}$, which constitutes about $40 \%$ of the operation time of the reference algorithm, with the accuracy at 97-98\% level in comparison to FEM. In addition, the time required to enter the input data and model the optimized disc must be taken into account. However, these steps can also be automated,

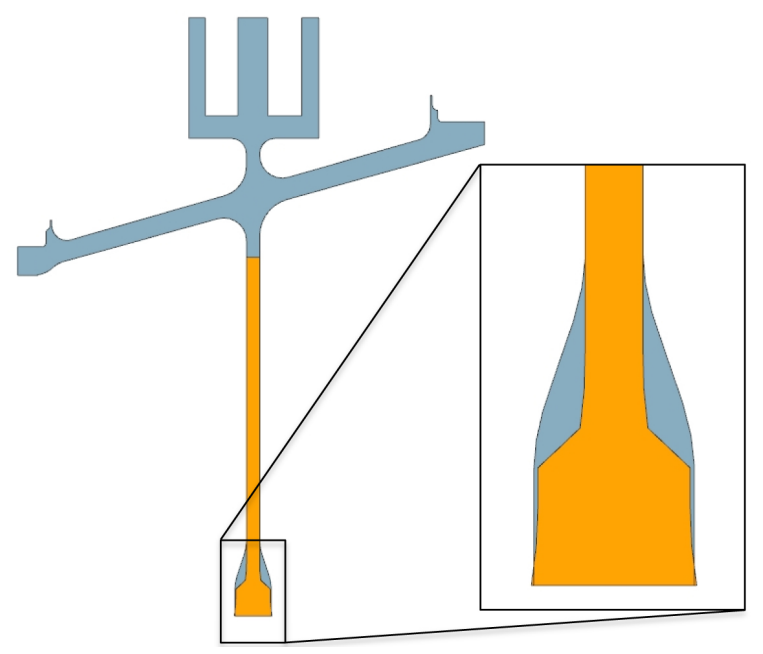

Fig. 10. Combination of profile of the output disc (grey) and optimized disc (orange). The area of the significant changes was zoomed for this reason, they were omitted as not affecting the absolute difference of the operation time between the reference algorithm and ANNs.

\section{CONCLUSIONS}

The proposed algorithm using the genetic algorithm and Artificial Neural Networks (ANNs) enables to reduce the time of optimization in a significant way. In the presented example of minimizing the mass of a compressor disc of the jet engine, after taking into account the ultimate strength condition due to ANNs implications, the operation time of the algorithm was reduced by $40 \%$ comparing to the reference algorithm. The reference genetic algorithm itself has been modified in comparison to the classical

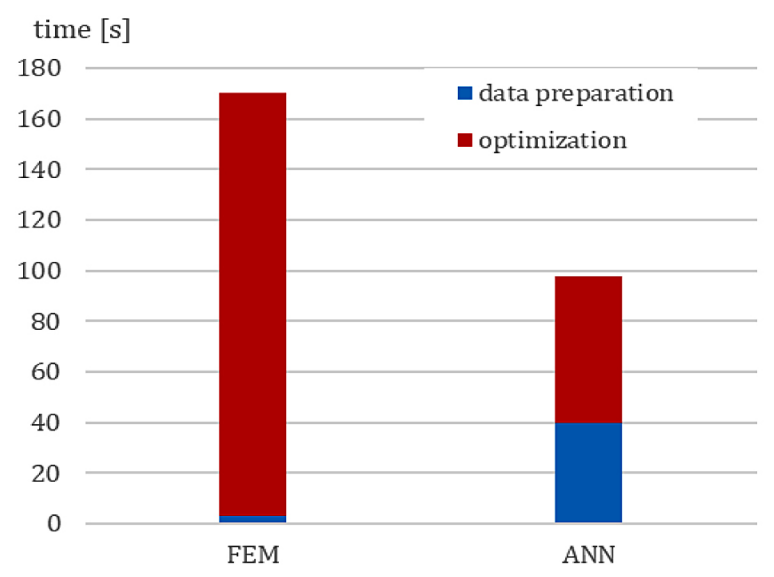

Fig. 11. Comparison of operation time of the algorithm based solely on FEM and the algorithm using ANNs 
literature-based approach through a variable area of acceptable solutions in each iteration. The application of such a modification of the genetic algorithm is acceptable if one is familiar with the general nature of the objective function or of an optimal solution.

The presented algorithm can be successfully applied wherever optimization is required, in case of which calculations of values of the objective function for any point are time-consuming. Some of these calculations can be successfully replaced with Artificial Neural Networks, even in the case when the differential equations are non-linear (to calculate the stress distribution in the rotating disc).

The aim of further research works will be to confirm that the proposed algorithm can also be used for other non-linear problems. The intention will be to replace the genetic algorithm with an algorithm based on self-learning neural networks, which (taking into account the gained experience) will be optimal to modify the shape of the disc by themselves. The algorithms based on such networks will be useful for optimizing typical objects, in case of which calculations of values of the objective function in the scope of decision variables would be extremely time consuming.

\section{REFERENCES}

1. Jahangir H. and Eidgahee A., New and robust hybrid artificial bee colony algorithm - ANN model for FRP-concrete bond strength evaluation, Composite Structures, 2021, 257.

2. Naderpour H., Rafiean A.H. and Fakharian, P., Compressive strength prediction of environmentally friendly concrete using artificial neural networks. Journal of Building Engineering, 2018, 16, 213-219.

3. Abdulla N.A., Using the artificial neural network to predict the axial strength and strain of concretefilled plastic tube. Journal of Soft Computing in Civil Engineering, 2020, 4(2), 63-86.

4. Naderpour H., Nagai K., Fakharian P. and Haji M., Innovative models for prediction of compressive strength of FRP-confined circular reinforced concrete columns using soft computing methods. Composite Structures, 2019, 215, 69-84.

5. Eidgahee D.R., Rafiean A.H. and Haddad, A., A novel formulation for the compressive strength of IBP-based geopolymer stabilized clayey soils using ANN and GMDH-NN approaches. Iranian Journal of Science and Technology, Transactions of Civil Engineering, 2020, 44(1), 219-229.

6. Pierret S. and Van den Braembussche R.A., Turbomachinery Blade Design Using a Navier-Stokes
Solver and Artificial Neural Network. J. Turbomach. 1999, 121(2), 326-332.

7. Roberts R. and Eastbourn S. Modeling techniques for a computational efficient dynamic turbofan engine model. International Journal of Aerospace Engineering. 2014.

8. Waszczyszyn Z. and Ziemiański L. Neural networks in mechanics of structures and materialsnew results and prospects of applications. Computers \& Structures, 2001, 79(22-25), 2261-2276.

9. Pabisek E. Systemy hybrydowe integrujące MES i SSN w analizie wybranych problemow mechaniki konstrukcji i materialow. Wydawnictwo Politechniki Krakowskiej, 2008.

10. Grzymowska A. Model neuronowy jako alternatywa dla numerycznego modelu okołodźwiękowego przepływu pary przez palisadę turbinową. Mechanik, 2014, 87, 217-224.

11. Głuch J. and Butterweck A. Zastosowanie sztucznych sieci neuronowych do diagnostyki cieplnoprzepływowej wieńców turbin parowych. Mechanik, 2014, 7, 173-180.

12. Koff L.B. Gas Turbine Technology Evolution: A Designers Perspective. Journal of Propulsion and Power. 2004, 20(4), 577-595.

13. Pierret S., Filomeno Coelho R. and Kato H. Multidisciplinary and multiple operating points shape optimization of three-dimensional compressor blades. Struct Multidisc Optim. 2007, 61-70.

14. Lee S.Y. and Kim K.Y. Design optimization of axial flow compressor blades with three-dimensional Navier-Stokes solver. KSME International Journal, 2000, 14(9), 1005-1012.

15. Panchenko V., Moustapha H., Mah S., Patel K. and Dowhan M. J. Preliminary multi-disciplinary optimization in turbomachinery design. Pratt and Whitney Canada Corp Longueuil (Quebec), 2003.

16. Abhishek S. and Goud B.N. Compressor Design optimization for a High speed Jet engine. IOP Conference Series: Materials Science and Engineering. 2018, 455, 1.

17. Schnoes M., Voß Ch. and Nicke E. Design optimization of a multi-stage axial compressor using throughflow and a database of optimal airfoils. Journal of the Global Power and Propulsion Society, 2018, 2, 516-528.

18. Kozakiewicz A. and Kieszek R. Zastosowanie sztucznych sieci neuronowych do obliczeń wytrzymałościowych maszyn wirnikowych. Przegląd Mechaniczny, 2018, LXXVII, 9, 28-30.

19. Nowotarski I. Obliczenia statyczne i dynamiczne turbinowych silników lotniczych metodą elementów skończonych. Wydawnictwa Naukowe Instytutu Lotnictwa, Warszawa 2001.

20. Møller M.F. A Scaled Conjugate Gradient Algorithm for Fast Supervised Learning. Neural Networks. 1993, 6, 525-533. 